\title{
DIABETES
}

\section{Have the gut(s) to test the risk of developing type 2 diabetes mellitus}

The risk of developing type 2 diabetes mellitus (T2DM) might be predicted by the gut metagenome, a new study suggests.

Previous studies have shown that the gut microbiota can affect host metabolism, and Chinese patients with T2DM have an altered gut metagenome. Now, Karlsson et al. extended these findings to a European population and examined whether the microbiota composition can identify diabetes status.

The researchers performed shotgun sequencing of DNA from faeces from 145 women aged 70 years ( 53 with T2DM, 49 with impaired glucose tolerance, 43 with normal glucose tolerance). "This [type of sequencing] provides us not only with taxonomical information but also functional insights, such as which microbial genes that are enriched in patients and controls", explains senior investigator Fredrik Bäckhed. The researchers then developed a model that can differentiate patients with T2DM from normal glucosetolerant individuals on the basis of the microbiota composition. Moreover, this model can classify prediabetic persons as either having metagenomes similar to healthy persons or those with T2DM.

The metagenomes found in the European population differed from that identified in Chinese individuals, but the functional alterations associated with T2DM were similar in the two populations.

"Future studies will reveal whether we can use the microbiota to predict the risk of developing T2DM and whether the gut microbiota may be a future therapeutic option", says Bäckhed.

Elisabeth Kugelberg

Original article Karlsson, F. H. et al. Gut metagenome in European women with normal, impaired and diabetic glucose control. Nature doi:10.1038/nature12198 